\title{
Blood glucose levels in adrenalectomized rats with an established sugar preference*
}

\author{
LESLIE E. FISHER, JR., Ohio University, Athens, Ohio 45710 \\ and
}

DOUGLAS L. GRIMSLEY, University of North Carolina, Charlotte, N.C. 28205

Blood glucose levels in rats with an established sugar preference were compared to rats with no such preference before and after adrenalectomy. The results indicated that an established preference for sugar does not affect the mill gram percentage of blood glucose under the present conditions. Regardless of the established preference, there were no significant differences between adrenalectomized groups for any of the postoperative periods.

In a recent report (Grimsley \& Fisher, 1967), it was shown that the development of a preoperative preference for a saccharine solution over a salt solution will not prevent the typical postoperative increase in salt drinking by adrenalectomized rats. Regardless of experience, adrenalectomized $S s$ drank less saccharine solution than sham Ss. These results were contrary to what would be predicted from Harriman (1955), who showed that an established preference for a sucrose over a salt solution would be maintained after adrenalectomy, to the detriment of the Ss. Grimsley \& Fisher (1967) hypothesized that Harriman's animals with an established sugar preference may have been satisfying a specific bodily need, namely, bood sugar level. Since it has been established that, under certain circumstances, hypoglycemia follows adrenalectomy (e.g., Long, Katzin, \& Fry, 1940), perhaps his animals were regulating body glucose levels instead of the electrolyte balance, which is usually considered to be of primary importance.

The present study was performed to see if there was a significant difference in blood glucose levels before and after adrenalectomy in rats with an established sugar preference compared to rats with no such preference.

SUBJECTS AND APPARATUS

The 108 male albino rats of the Dublin strain (Dublin, Virginia) were approximately 90 days old at the start of experimentation.

A Na-deficient diet (Hartroft formula, General Biochemicals) was available to all animals ad lib. It was placed in a standard foodcup at the rear of the cage and filled daily.

*Supported in part by National Science Foundation Grant No.GY 2565 to West $V$ irginia State College and by Obio University. The authors gratefully acknowledge the assistance of William Hicks in animal maintenance and data collection.
The test solutions consisted of a $1.5 \% \mathrm{NaCl}$ and $8.4 \%$ sucrose solution, modeled after Harriman (1955). Solutions were prepared with reagent grade chemicals, using distilled water as the solvent, and were mixed on a weight/volume basis. The solutions were presented in calibrated bottles with stainless steel drinking tubes which protruded into the cage about 3 in. apart. To control for position effect and preference for a particular drinking tube, the method of Bare (1949) was used: each solution was presented in each position in each bottle.

\section{PROCEDURE}

The 108 Ss were divided randomly into two conditions. Food and liquids were freely available, and intake was recorded daily.

\section{Condition 1}

Groups $A$ and $B(N=27$ each $)$ received both sucrose and $\mathrm{NaCl}$ during the 15 preoperative days (experience groups). On Day 1, blood samples were taken preoperatively from three animals from each group by cardiac puncture and the animals were sacrificed. Samples continued to be taken preoperatively from three animals from each group on Days 5 , 10 , and 15. On Day 16, the animals of Group $A$ were bilaterally adrenalectomized and those of Group B were bilaterally sham adrenalectomized. (In the sham operation, the adrenal glands were exposed but not removed.) Animals in both groups continued to receive the sucrose and $\mathrm{NaCl}$ solutions postoperatively. Three animals from each group were sacrificed to obtain blood samples on Days 1, 5, 10, 15, and 20 , postoperatively.

\section{Condition 2}

Groups $\mathrm{C}$ and $\mathrm{D} \quad(\mathrm{N}=27$ each $)$ received only distilled water during the preoperative period (no-experience groups). Three animals from each group provided blood for glucose analysis preoperatively on Days 1,5 ,
10 and 15 and on Days $1,5,10,15$ and 20 , postoperatively. On the 16 th day, the 15 animals remaining in Group 3 were adrenalectomized and the 15 animals in Group 4 were sham operated. Both groups were given postoperative access to the sucrose and $\mathrm{NaCl}$ solutions. Postmortem verification of adrenalectomy was gained following collection of blood samples.

Blood sugar determinations were made by the Hycel P-M-S Reagent Method (Hycel Chemical Co., Texas). RESULTS

The blood sugar data, in milligram percent, from the three animals from the four groups on each of the preoperative and postoperative days sampled were compared by analysis of variance procedures. There were no significant differences between groups on any of the preoperative days $(p>.05)$. Furthermore, there were no significant differences between groups on Days 1, 5, 10, and 15, postoperatively $(p>.05)$. On Postoperative Day 20, however, there was a significant difference $(p<.01)$ in blood glucose levels between groups $(\mathrm{F}=12.9, \mathrm{df}=3 / 8)$. Further analysis by the Newman-Keuls procedure indicated that adrenalectomized animals had less blood sugar than the sham animals, though the adrenalectomized groups did not differ from each other, and nor were the sham groups different from each other.

\section{DISCUSSION}

There were no differences in blood glucose levels between experienced and no-experience animals for any of the preoperative or postoperative periods: An established preference for sugar does not affect the milligram percentage of blood glucose under the present conditions. The groups were similar, despite the preoperative experience.

The only significant difference obtained was between the sham and adrenalectomized groups on Day 20. The findings are consistent with the results from Long et al (1940). While the blood sugar levels of adrenalectomized animals were consistently below those of the sham animals, it was only on Day 20 that this difference reached significance.

Since the blood glucose levels were not significantly different for experienced and no-experience animals for any of the postoperative days measured, it appears unlikely that the data previously reported by Harriman (1955) can be attributed to blood sugar regulation. Animals with an established preference for glucose do not maintain a blood glucose level different from animals with no such experience. Therefore, it appears that 
regulation of blood glucose levels, instead of electrolyte balance, as hypothesized by Grimsley \& Fisher (1967), will not explain satisfactorily the unique results reported by Harriman (1955); his data remain the only report of a failure of rats to seek salt following adrenalectomy.

\section{REFERENCES}

BARE, J. K. The specific hunger for sodium chloride in normal and adrenalectomized rats. Journal of Comparative \& Physiological Psychology, 1949, 42. 242-253.

GRIMSLEY, D. L., \& FISHER, L. E. Preoperative saccharine experience and postoperative fluid preference in adrenalectomized rats. Psychonomic Science, 1967, 9, 299-300.

HARRIMAN, A. E. The effect of a preoperative perference for sugar over salt upon compensatory salt selection by adrenalectomized rats. Journal of Nutrition, 1955, 57, 271-276.

LONG, C. N.. KATZIN. B., \& FRY, E. G. The adrenal cortex and carbohydrate metabolism. Endocrinology, 1940, 26, 309-344.

\section{The effect of pretraining on schedule induced polydipsia*}

\author{
F. M. TOATES† \\ Laboratory of Experimental Psychology \\ University of Sussex, Brighton, England
}

In order to evaluate superstition and thirst explanations of psychogenic polydipsia, thirsty rats were allowed water from a burette in a test box. Later, when hungry, they were given food pellets on a 90-sec free fixed-interval schedule (FFI). This procedure was followed during daily 3-h sessions for 14 days, water being available in the home cage but not in the test box. They were then subjected to the same schedule but with water available during sessions. Their behavior with water available was compared to that of a control group which had not received the pretraining without water. The group which had been pretrained showed a retardation in their acquisition of polydipsia.

If a rat which is food deprived but not water deprived obtains food pellets on an interval schedule (by barpress or free delivery) and if a waterspout is in the delivery apparatus, relatively enormous quantities of water will be drunk (Falk, 1961). The animal drinks immediately after each food pellet (Burks, Hitzing, \& Schaeffer, 1967). A strong controversy surrounds the

*This work was carried out while the author was supported by a grant from the SRC.

+I am very grateful to Dr. K. Oatley for his supervision of and assistance in this project. explanation of the phenomenon: Stein (1964) argues that it represents a form of thirst; others (e.g., Clark, 1962) argue that it is an example of adventitious reinforcement or superstition. The strongest argument against the superstition hypothesis is the timing of the drinking, since it always follows, rather than precedes, food delivery. Recently, Burks (1970) concluded that the effect was under the control of factors other than adventitious food reinforcement. that, even if drinking delayed food delivery, polydipsia still appeared. Hymowitz \& Koronakos (1968) Similarly, Segal \& Oden (1969) found showed that a history of obtaining water and food always at separate times played no significant part in the development of polydipsia. Recently, an extensive review of the subject appeared (Falk, 1969).

The present experiment was designed to investigate the effect of previous experience upon the acquisition of polydipsia. If the drinking is in response to thirst produced by eating dry food, then experience in a test box of obtaining both water when food is absent and food when water is absent should, when both are available together, encourage the rapid appearance of polydipsia, On a thirst explanation, it might be argued that the reason polydipsia takes time to develop is that the animal's thirst is secondary to its hunger, and, therefore, activity is primarily directed to obtaining food. The interval schedule is such that, as the trials proceed, the animal learns that it can generally afford to be away from the food delivery area immediately after the delivery of a pellet with no harmful effect as far as obtaining food is concerned. The magnitude of the amount drunk during interval schedules may be explained by a transient dry-mouth thirst. Intermittent delivery schedules give full opportunity for this to be relieved, while under ad lib eating conditions the continuation of eating takes priority. The experiment was, therefore, primarily designed to allow early appearance of polydipsia, should the explanation in terms of thirst be true.

In contrast, if the drinking represents superstitious behavior, then allowing the animal to obtain food for some length of time, without association with drinking, should discourage a superstitious association from being formed or at least retard its development.

\section{METHOD}

The Ss were 14 male hooded rats of a mean weight of $366 \mathrm{~g}$ before deprivation. In their home cages, they had obtained water from a burette like the one in the test box. The test apparatus consisted of a metal box, $20 \times 18 \times 14 \mathrm{~cm}$, with a lamp built into the roof. At one end was the food delivery tray and $10 \mathrm{~cm}$ away, when available, was the spout of a burette containing water. The food pellets were of commercial rat chow (Diet 41B obtained from Bradley and Bliss) and each weighed $54 \mathrm{mg}$. In the home cage, Spillers' Small Animal Diet was supplied to the extent necessary to maintain weight. All animals were run at $80 \%$ of their predeprivation body weights. The amount of water drunk was measured to the nearest $0.1 \mathrm{ml}$. 